\title{
What Does Local Functional Hyperemia Tell about Local Neuronal Activation?
}

\author{
Natalya Jukovskaya, ${ }^{1,2,3}$ Pascale Tiret, ${ }^{1,2,3}$ Jérôme Lecoq, ${ }^{1,2,3}$ and Serge Charpak ${ }^{1,2,3}$ \\ ${ }^{1}$ Institut National de la Santé et de la Recherche Médicale, U603, ${ }^{2}$ Centre National de la Recherche Scientifique, UMR 8154, and ${ }^{3}$ Laboratory of \\ Neurophysiology and New Microscopies, Université Paris Descartes, Paris 75006, France
}

In the brain, neuronal activation triggers a local increase in cerebral blood flow, a response named functional hyperemia. The extent to which functional hyperemia faithfully reports brain activation, spatially or temporally, remains a matter of debate. Here, we used the olfactory bulb glomerulus as a neurovascular model and two-photon microscopy imaging to investigate the correlation between calcium signals in glutamatergic terminals of olfactory sensory neurons and local vascular responses. We find that, depending on odor stimulation intensity, vascular responses are differently coupled to calcium signals. Upon moderate odor stimulation, glomerular vascular responses increase accordingly with calcium signals. In contrast, in silent glomeruli neighboring strongly activated ones and in glomeruli adapting upon high odor stimulation, vascular responses are independent of or negatively coupled to presynaptic calcium signals, respectively. Hence, functional hyperemia, a key signal used in functional imaging, can be, at times, an unreliable marker of local brain activation.

\section{Introduction}

In humans, most noninvasive brain imaging techniques assess neuronal activation from measurement of hemodynamic changes. However, if functional hyperemia is indeed tightly correlated to neuronal activation, the molecular and cellular mechanisms underlying this neurovascular coupling are not fully understood (for review, see Gordon et al., 2007; Iadecola and Nedergaard, 2007; Attwell et al., 2010). There is now a general consensus that neurons increase cerebral blood flow (CBF) using a feedforward mechanism in which input activity leads to the release of glutamate and adenosine triphosphate, followed by the activation of several signaling pathways involving astrocytes and neurons, and controlling the arteriolar tone. The multiplicity of these signaling pathways raises the question of the extent to which $\mathrm{CBF}$ changes and blood oxygen level-dependent (BOLD) functional magnetic resonance imaging (fMRI) signals reflect local neuronal activity. Both signals are best correlated to local field potential (LFP) responses rather than to spiking (Logothetis, 2008) and are maintained in conditions where spiking is strongly reduced (Mathiesen et al., 1998; Rauch et al., 2008). Still, LFP responses themselves are complex and involve EPSPs, IPSPs, neuronal intrinsic membrane properties, and occasionally volume-conducted LFP responses from neighboring activated networks. Therefore, it is questionable that LFP responses always faithfully reflect the level of glutamatergic input activation, which should be best represented by measurements of glutamate release.

Received June 18, 2010; revised Nov. 9, 2010; accepted Nov. 30, 2010.

This work was supported by the Leducq Fundation, the Fondation Bettancourt Schueller, the Human Frontier Science Program Organization, the European Commission FP6 (LSHM-CT-2007-037765), and the Fondation pour la Recherche Médicale. We thank Jonathan Bradley and Etienne Audinat for their critical comments.

Correspondence should be addressed to Serge Charpak, Institut National de la Santé et de la Recherche Médicale U603, Laboratory of Neurophysiology, 45 rue des St Pères, 75006 Paris, France. E-mail: serge. charpak@parisdescartes.fr.

DOI:10.1523/JNEUROSCI.3146-10.2011

Copyright $\odot 2011$ the authors $\quad 0270-6474 / 11 / 311579-04 \$ 15.00 / 0$
The functional organization of olfactory bulb glomeruli make them ideal functional modules to investigate neurovascular coupling and functional hyperemia (Shepherd and Charpak, 2008). During odor stimulation, LFP responses have several components; one of them, the LFP rapid negativities, reflects neuronal activation restricted to a single glomerulus. These negativities are odor-specific, locked to the respiration frequency [which is driving air past the olfactory receptor neurons (ORNs)], show sharp transitions in space (laterally and in depth), are tightly correlated to mitral cell postsynaptic activity, and are abolished by intraglomerular application of glutamate ionotropic receptor antagonists (Chaigneau, 2007). LFP negativities thus reflect the summation of EPSP in glomeruli. More recently, we have labeled ORN terminals with the calcium sensor, calcium green 1 (Lecoq et al., 2009), and shown that LFP rapid negativities are tightly correlated to presynaptic calcium $\left(\mathrm{Ca}^{2+}\right)$ signals detected in ORN terminals converging into the same glomerulus. This correlation is surprisingly strong and independent of odor stimulation intensity, suggesting that presynaptic $\mathrm{Ca}^{2+}$ signals could be considered as solid markers of the amount of glutamate released onto the glomerular dendrites that generate LFP negativities. Because odors also trigger blood flow increases in glomeruli, a response termed glomerular functional hyperemia (Kida et al., 2002; Chaigneau et al., 2003, 2007; Petzold et al., 2008; Shepherd and Charpak, 2008), we examined whether this functional hyperemia is correlated to presynaptic $\mathrm{Ca}^{2+}$ signals in ORN terminals under three conditions of neuronal activation: in the absence or presence of peripheral adaptation and below the ORN activation threshold.

\section{Materials and Methods}

Animal preparation and two-photon imaging. Wistar rats, 30-60 d old, were anesthetized with an intraperitoneal bolus injection of ketamine and xylazine ( 90 and $10 \mathrm{mg} / \mathrm{kg}$, respectively) followed by a continuous intravenous injection (25-30\% of the bolus/h). The animal was held in a 
standard stereotaxic apparatus with ear bars and a craniotomy was performed above the two olfactory bulb hemispheres, the posterior cisterna was drained, and the dura was removed. A $100-\mu$ m-thick glass coverslip was placed over the bulb, fixed on the cranium and the space below filled with a 3\% Agar solution. The temperature of the animal was monitored with a rectal thermometer and maintained at $37^{\circ} \mathrm{C}$ with a feedback controlled heating blanket (Harvard Apparatus). For all experiments, breathing frequency was monitored through a pneumogram transducer (BIOPAC Systems). Heart rate, oxygen saturation, and pulse distension were monitored through a pulse oxymeter located on the rat hindpaw (STARR Life Sciences). Animals continuously breathed filtered air enriched with oxygen (20-30\%). Olfactory nerve terminals were labeled with $\mathrm{Ca}^{2+}$ green 1 dextran, $10 \mathrm{kDa}$ (Invitrogen), 2-8 d before experiments, as described previously (Lecoq et al., 2009). To label vessels, a bolus of $70 \mathrm{kDa}$ Texas Red dextran was injected intravenously through a catheter placed in the femoral vein. $\mathrm{Ca}^{2+}$ measurements were obtained either from images comprising the entire glomerulus and acquired at rates up to 20 frames per second (using a custom-built twophoton laser-scanning microscope) or from line scans running through a capillary and the neighboring neuropil (Chaigneau et al., 2007). $\mathrm{Ca}^{2+}$ changes and red blood cell (RBC) velocity were measured as previously described (Chaigneau et al., 2007). Note that, because $\mathrm{Ca}^{2+}$ changes $(\Delta \mathrm{F} / \mathrm{F})$ obtained with movies and line scans were similar, all data are pooled in the statistics. The initial $\mathrm{Ca}^{2+}$ peak or the overall $\mathrm{Ca}^{2+}$ signal (area) was used for quantification, as indicated in the text. For the entire study, average values are expressed as mean \pm SEM.

Odor delivery. Odorants were applied for $4 \mathrm{~s}$ with a custom-built olfactometer. The olfactometer delivered a constant flow of humidified air in a small Teflon reservoir surrounding the rat snout. All tubes connecting the odorant reservoir to the snout were made of Teflon to minimize odorant contamination. Odorants used were aldehydes and esters. Two flow meters (Aalborg), one in the clean air path and one before the odorant reservoir, allowed us to determine precisely the odorant concentration. To reveal adaptation, high odorant concentrations were used, i.e., from $3-100 \%$ when expressed in percentage of saturated air. Odor applications were repeated at least three times (with 3-4 min intertrial intervals) at each odorant concentration.

\section{Results}

We first determined that, in glomeruli that do not show peripheral adaptation during a sustained odor stimulation (4s), the overall presynaptic $\mathrm{Ca}^{2+}$ signals and vascular responses increased accordingly with the odor stimulation intensity (Fig. $1 A, B)$. This was expected from our previous quantifications based on the summed LFP peaks ( $\Sigma$ LFP) (Chaigneau et al., 2007) and is in accordance with what has been reported in many brain concentration.
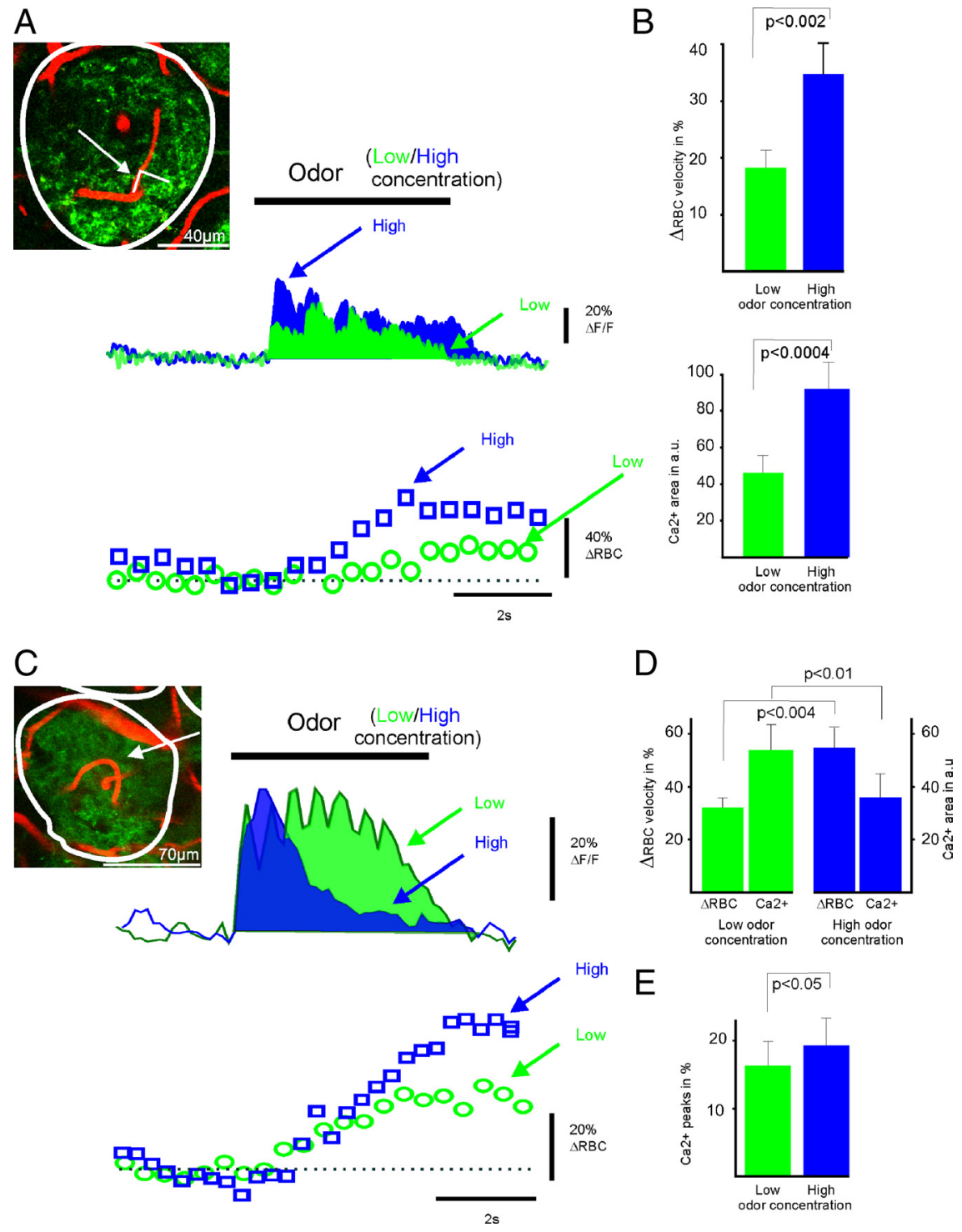

Figure 1. Functional hyperemia and glutamate release by ORN terminals in the absence and presence of peripheral odor adaptation. $\boldsymbol{A}$, Neuronal and vascular responses in the absence of odor adaptation. Top left, Glomerulus with boundaries outlined by ORN terminals labeled with calcium $\left(\mathrm{Ca}^{2+}\right)$ green dextran, and a capillary (arrow) in which RBC velocity was monitored. Line scans were acquired on a segment going along the longitudinal axis of the capillary for its first part and through the neighboring neuropil for its second part. RBC velocity measurements (the first part of the line scan) were acquired on one detector and $\mathrm{Ca}^{2+}$ on a second one. Top scan, At low concentration, ethyl butyrate (50\%; green trace) induced a presynaptic $\mathrm{Ca}^{2+}$ signal, which overall response (the area below the curve, in a.u.) increased with concentration (high $=75 \%$; blue trace). Bottom scan, The vascular response increased accordingly with the odor concentration. $B$, Summary graphs showing that functional hyperemia is positively coupled to the overall $\mathrm{Ca}^{2+}$ signal ( $n=9$ capillaries in seven animals). $C$, Neuronal and vascular responses in the presence of peripheral adaptation. Top left, The recorded glomerulus and capillary. $\mathrm{Ca}^{2+}$ responses were measured over the entire glomerulus at a rate of 9 image/s. Top scan, At low odorant concentration (heptaldehyde, 17\%), the presynaptic $\mathrm{Ca}^{2+}$ response displayed a large initial $\mathrm{Ca}^{2+}$ signal peak followed by smaller ones which were phase-locked to respiration and maintained during the entire stimulus duration. At higher odorant concentration (83\%), the initial $\mathrm{Ca}^{2+}$ peak was slightly larger; however, the overall $\mathrm{Ca}^{2+}$ response markedly decreased (blue trace). Bottom scan, The vascular response was larger at high than low odor concentration. $\boldsymbol{D}$, $\boldsymbol{E}$, Summary graphs showing that during odor adaptation ( $n=9$ animals), functional hyperemia is positively coupled to the initial presynaptic $\mathrm{Ca}^{2+}$ signal, i.e., glutamate released during the first inhalation but negatively coupled to the overall $\mathrm{Ca}^{2+}$ signal, i.e., glutamate continuously released during the sustained stimulation. Averages of three to six odor applications at each odor

regions upon moderate glutamatergic input activation (Iadecola et al., 1996; Mathiesen et al., 1998; Norup Nielsen and Lauritzen, 2001; Logothetis et al., 2001). In contrast, the correlation between presynaptic $\mathrm{Ca}^{2+}$ signals and vascular responses markedly differed in more sensitive glomeruli showing peripheral ORN adaptation. 
A

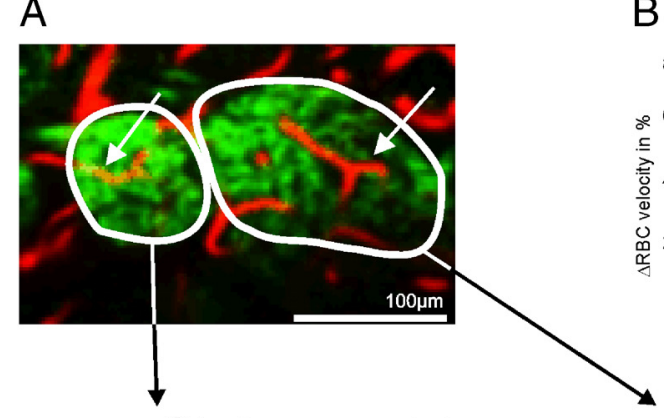

Odor (Low concentration)

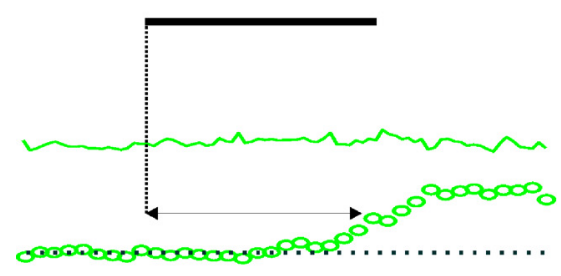

Odor (High concentration)

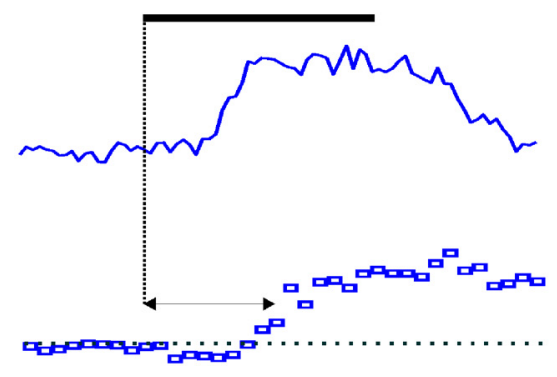

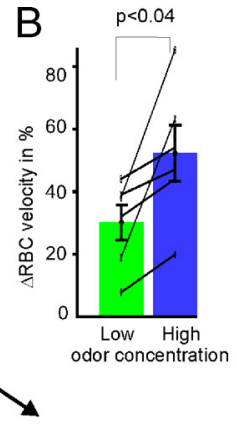

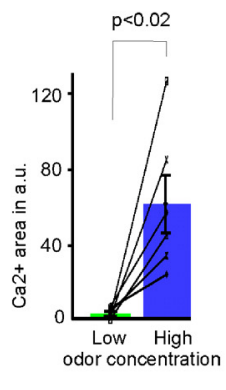

Odor (Low concentration)

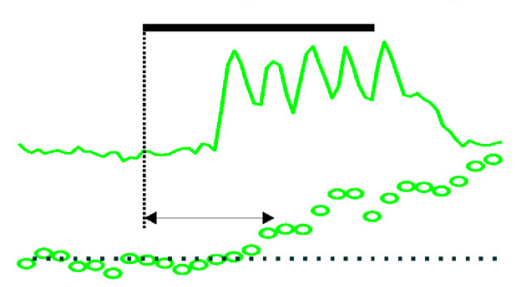

Odor (High concentration)

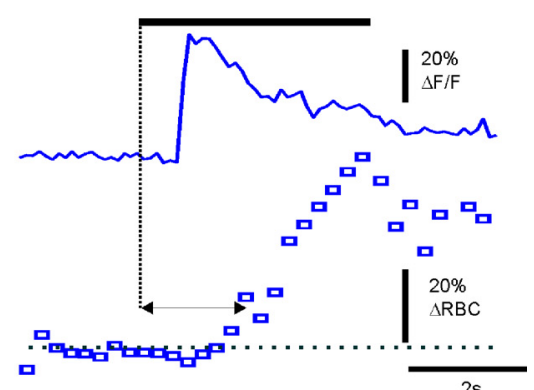

2s

Figure 2. Functional hyperemia below the ORN activation threshold. $\boldsymbol{A}$, Top left, Two glomeruli in which presynaptic $\mathrm{Ca}^{2+}$ responses were simultaneously imaged and in which $\mathrm{RBC}$ velocity was sequentially measured in capillaries indicated by the arrows. Middle left, At low concentration, heptaldehyde (16\%) did not cause any $\mathrm{Ca}^{2+}$ response; however, it triggered a delayed RBC velocity increase. The horizontal double-headed arrow indicates the delay between the odor onset and the vascular response. Middle right, In contrast, the same concentration induced typical presynaptic $\mathrm{Ca}^{2+}$ and vascular responses in the adjacent glomerulus. Bottom, At higher concentrations (100\%), heptaldehyde stimulated both glomeruli. Note that the vascular delay of the left glomerulus significantly decreased. Averages of three or four odor applications at each odor concentration. $\boldsymbol{B}$, Summary graphs showing that, in glomeruli adjacent to strongly responsive glomeruli, functional hyperemia occurs in the absence of presynaptic $\mathrm{Ca}^{2+}$ signal, i.e., in the absence of glutamate locally released (mean \pm SEM and responses of individual capillaries, $n=6$ animals).

Such adaptation is triggered by $\mathrm{Ca}^{2+}$ influx in ORNs in the epithelium, and involves several negative feedback processes, among which is a sensitivity decrease of cyclic nucleotide-gated channels or the hydrolysis of cAMP (for review, see Kleene, 2008). We have recently shown that peripheral adaptation can also be detected centrally in glomeruli, with LFP responses decreasing upon strong odorant stimulation (Lecoq et al., 2009). This phenomenon did not involve postsynaptic processing but resulted from a decrease of presynaptic $\mathrm{Ca}^{2+}$ signals in ORN terminals and thus a decrease of the amount of glutamate released by these terminals. Because presynaptic GABA B receptors (Aroniadou-Anderjaska et al., 2000; Murphy and Isaacson, 2003; Vucinic et al., 2006; Pírez and Wachowiak, 2008), dopamine receptors (Hsia et al., 1999), or purinergic receptors (our unpublished observations) were not involved in the phenomenon (Lecoq et al., 2009), we could conclude that presynaptic $\mathrm{Ca}^{2+}$ signal adaptation indirectly reflected peripheral adaptation. Figure 1, $C$ and $D$, illustrates that, during such peripheral adaptation, the overall presynaptic $\mathrm{Ca}^{2+}$ signal from ORNs and the vascular response uncoupled; during strong odor stimulation (Fig. 1C, blue), the local vascular response was larger, whereas the overall presynaptic $\mathrm{Ca}^{2+}$ signal was smaller than during weak stimulation (Fig. $1 C$, green). In nine animals (Fig. 1D), the mean change in RBC velocity increased from $31 \pm 3 \%$ to $54 \pm$ $7 \%$ when the odor concentration increased, leading to peripheral adaptation, whereas the overall presynaptic $\mathrm{Ca}^{2+}$ signal decreased from $53 \pm 9$ [arbitrary units (a.u.)] to $35 \pm 9$ (a.u.). However (Fig. 1 E), a positive correlation was maintained between the initial $\mathrm{Ca}^{2+}$ peak and vascular responses during strong stimulation. These data show that during peripheral adaptation, functional hyperemia is coupled in a complex manner to locally presynaptic $\mathrm{Ca}^{2+}$. During sustained odor stimulation, the vascular response is correlated in a concentration-dependent manner to the first $\mathrm{Ca}^{2+}$ transient, i.e., the first inhalation, but then becomes progressively independent of, or even negatively coupled to, the over $\mathrm{Ca}^{2+}$ rise. We propose that during peripheral adaptation, functional hyperemia reports the regional and not local glomerular activation (see Discussion, below).

In a second series of experiments, we investigated whether functional hyperemia could be detected in the absence of glutamate release, i.e., below the ORN activation threshold. Glomeruli were precisely chosen, with one weakly sensitive, responding only during strong stimulation and adjacent to a second glomerulus, which responded during weak stimulation and eventually adapted at higher odor concentration. Figure 2 shows that in the weak glomerulus immediately adjacent to the strongly responsive glomerulus and in the absence of a presynaptic $\mathrm{Ca}^{2+}$ increase, vascular responses, although delayed, could be recorded (mean $\triangle \mathrm{RBC}$ velocity increase, $30 \pm 6 \%$ ). Note that in the weak glomerulus, increasing the odor concentration generated specific and local $\mathrm{Ca}^{2+}$ increases (mean $\Delta \mathrm{Ca}^{2+}$ of $62 \pm 16$ a.u.) and vascular responses (mean $\triangle \mathrm{RBC}$ velocity increase, $52 \pm 9 \%$ ), demonstrating that ORN terminals were functional. Note that the vascular response latency decreased at higher odor concentration (from $3.9 \pm 0.6$ to $2.0 \pm 0.3 \mathrm{~s} ; n=6, p=0.045)$. These results show that strongly activated glomeruli may influence blood flow in neighboring inactive glomeruli, demonstrating that due to the loose vascular tree organization (e.g., in the presence of capillaries crossing directly from one glomerulus to another), the spatial resolution of vascular responses is not as sharp as that of neuronal responses.

\section{Discussion}

Glomeruli are particularly well defined neuronal networks. However, even in such a simple neuronal model, the relationship between functional hyperemia and neuronal activation is complex. Under moderate odor stimulation, i.e., in the absence of adaptation, vascular responses and postsynaptic responses ( 2 LFP) 
(Chaigneau et al., 2007) or presynaptic $\mathrm{Ca}^{2+}$ signals are positively correlated. This is in agreement with numerous studies reporting strong correlations between LFP and BOLD fRMI or laser Doppler signals (for review, see Logothetis, 2008). However, at a higher odor concentration, functional hyperemia no longer reflects the level of local sensory stimulation measured from markers such as $\Sigma$ LFP peaks or the overall presynaptic $\mathrm{Ca}^{2+}$ responses. Note that such mismatch between vascular responses and presynaptic $\mathrm{Ca}^{2+}$ would have been similarly observed if glomerular adaptation was due to presynaptic inhibition and not to ORN adaptation. Finally, the presence of a delayed nonspecific vascular response in glomeruli adjacent to activated glomeruli also stresses that the vascular tree organization does not allow vascular signals to perfectly match neuronal activity.

Can we extend our findings, exclusively obtained in olfactory glomeruli, to other brain regions? The strength of the glomerular model, as opposed to other regions where some neurovascular uncoupling has been suggested (Norup Nielsen and Lauritzen, 2001; Kim et al., 2004), is that it is a unique structure in which glutamate release from ORN terminals can be inferred from presynaptic $\mathrm{Ca}^{2}$ and modulated physiologically and in a quantified manner. The strong correlation between LFP peaks and $\mathrm{Ca}^{2+}$ transients justify such a hypothesis, in particular since in vitro, the relationship between ORN transmitter release and extracellular calcium is almost linear (Murphy et al., 2004). In fact, the overall $\mathrm{Ca}^{2}$ is probably the best marker of glutamate release, as opposed to the overall LFP response that comprises nonsynaptic and volume-conducted components. We conclude that if vascular responses are to be generally considered well correlated to glutamate release and subsequent neuronal activation, credence should be paid to their presence or absence in particular conditions of neuronal network activation such as adaptation of any glutamatergic inputs or presynaptic inhibition. Thus, functional hyperemia and BOLD fMRI signals, under all kinds of brain activity, are not a priori read outs of local neuronal activation.

\section{References}

Aroniadou-Anderjaska V, Zhou FM, Priest CA, Ennis M, Shipley MT (2000) Tonic and synaptically evoked presynaptic inhibition of sensory input to the rat olfactory bulb via GABA(B) heteroreceptors. J Neurophysiol 84:1194-1203.

Attwell D, Buchan AM, Charpak S, Lauritzen M, Macvicar BA, Newman EA (2010) Glial and neuronal control of brain blood flow. Nature 468:232-243.

Chaigneau E, Oheim M, Audinat E, Charpak S (2003) Two-photon imaging of capillary blood flow in olfactory bulb glomeruli. Proc Natl Acad Sci U S A 100:13081-13086.
Chaigneau E, Tiret P, Lecoq J, Ducros M, Knöpfel T, Charpak S (2007) The relationship between blood flow and neuronal activity in the rodent olfactory bulb. J Neurosci 27:6452-6460.

Gordon GR, Mulligan SJ, MacVicar BA (2007) Astrocyte control of the cerebrovasculature. Glia 55:1214-1221.

Hsia AY, Vincent JD, Lledo PM (1999) Dopamine depresses synaptic inputs into the olfactory bulb. J Neurophysiol 82:1082-1085.

Iadecola C, Nedergaard M (2007) Glial regulation of the cerebral microvasculature. Nat Neurosci 10:1369-1376.

Iadecola C, Li J, Xu S, Yang G (1996) Neural mechanisms of blood flow regulation during synaptic activity in cerebellar cortex. J Neurophysiol 75:940-950.

Kida I, Xu F, Shulman RG, Hyder F (2002) Mapping at glomerular resolution: fMRI of rat olfactory bulb. Magn Reson Med 48:570-576.

Kim DS, Ronen I, Olman C, Kim SG, Ugurbil K, Toth LJ (2004) Spatial relationship between neuronal activity and BOLD functional MRI. Neuroimage 21:876-885

Kleene SJ (2008) The electrochemical basis of odor transduction in vertebrate olfactory cilia. Chem Senses 33:839-859.

Lecoq J, Tiret P, Charpak S (2009) Peripheral adaptation codes for high odor concentration in glomeruli. J Neurosci 29:3067-3072.

Logothetis NK (2008) What we can do and what we cannot do with fMRI. Nature 453:869-878.

Logothetis NK, Pauls J, Augath M, Trinath T, Oeltermann A (2001) Neurophysiological investigation of the basis of the fMRI signal. Nature 412:150-157.

Mathiesen C, Caesar K, Akgören N, Lauritzen M (1998) Modification of activity-dependent increases of cerebral blood flow by excitatory synaptic activity and spikes in rat cerebellar cortex. J Physiol 512:555-566.

Murphy GJ, Isaacson JS (2003) Presynaptic cyclic nucleotide-gated ion channels modulate neurotransmission in the mammalian olfactory bulb. Neuron 37:639-647.

Murphy GJ, Glickfeld LL, Balsen Z, Isaacson JS (2004) Sensory neuron signaling to the brain: properties of transmitter release from olfactory nerve terminals. J Neurosci 24:3023-3030.

Norup Nielsen A, Lauritzen M (2001) Coupling and uncoupling of activitydependent increases of neuronal activity and blood flow in rat somatosensory cortex. J Physiol 533:773-785.

Petzold GC, Albeanu DF, Sato TF, Murthy VN (2008) Coupling of neural activity to blood flow in olfactory glomeruli is mediated by astrocytic pathways. Neuron 58:897-910.

Pírez N, Wachowiak M (2008) In vivo modulation of sensory input to the olfactory bulb by tonic and activity-dependent presynaptic inhibition of receptor neurons. J Neurosci 28:6360-6371.

Rauch A, Rainer G, Logothetis NK (2008) The effect of a serotonin-induced dissociation between spiking and perisynaptic activity on BOLD functional MRI. Proc Natl Acad Sci U S A 105:6759-6764.

Shepherd GM, Charpak S (2008) The olfactory glomerulus: a model for neuro-glio-vascular biology. Neuron 58:827-829.

Vucinić D, Cohen LB, Kosmidis EK (2006) Interglomerular centersurround inhibition shapes odorant-evoked input to the mouse olfactory bulb in vivo. J Neurophysiol 95:1881-1887. 